\title{
Parametric Wavelength Conversion From Conventional Near-Infrared to Visible Band
}

\author{
R. Jiang, R. Saperstein, N. Alic, M. Nezhad, C. McKinstrie, J. Ford, Y. Fainman, and S. Radic
}

\begin{abstract}
The feasibility of distant wavelength conversion in photonic crystal fiber was explored. The one-pump parametric architecture was used to demonstrate translation from the conventional $(1550 \mathrm{~nm})$ to visible $(500 \mathrm{~nm})$ band. Experimental results are reported, which demonstrate the conventional-to-visible conversion of single- and multiple-channel signals encoded in the nonreturn-to-zero format.
\end{abstract}

Index Terms-Band translation, four-photon mixing, parametric process.

\section{INTRODUCTION}

$\mathbf{T}$ HE conventional 1550-nm band is used almost exclusively for optical transport, to support both high-capacity and long-reach applications. The choice of the 1550-nm spectral window was originally motivated by low fiber loss and the introduction of optical amplifiers. Its dominance was later reinforced by many advanced technologies that presently do not exist in other spectral windows. Indeed, no erbium-doped fiber amplifier (EDFA) equivalent exists in the visible or the infrared (IR) bands; complex phase or amplitude modulation is rarely possible outside the 1550 -nm band and fast 1550 -nm receivers are superior to those in other bands. However, critical applications exist outside the 1550 -nm band, and include submarine or airborne transport, spectrally invariant sensing, and spectroscopy. Instead of developing new band-specific technologies, the construction of a universal band translator (UBT) would allow the use of mature 1550-nm technology in other spectral windows. The general scheme consists of modulation in the 1550-nm band, translation to the target (transport) window, and, finally, reverse translation to the 1550-nm band for reception and decoding.

Parametric processes are excellent candidates for a spectrally invariant UBT technology extending from the visible to the IR. A candidate process, $\chi^{(3)}$ photon exchange in silica offers subpicosecond response and strict phase and quantum state preservation over a wide spectral range [1], [2]. Specifically, high-confinement optical fibers are identified for their high efficiencies and wide bandwidths when used in single-pass traveling architectures. Parametric processing in fiber has been revitalized by recent advances in the manufacturing of highly nonlinear fiber (HNLF). HNLF offers parametric amplification and conversion efficiencies [1], [2] in excess of $40 \mathrm{~dB}$ in the immediate vicinity of the $1550-\mathrm{nm}$ band. Unfortunately, HNLF is a classical step-index fiber design that does not support monomode

Manuscript received April 21, 2006; revised July 28, 2006.

R. Jiang, R. Saperstein, N. Alic, M. Nezhad, J. Ford, Y. Fainman, and S. Radic are with the University of California San Diego, La Jolla, CA 92093-0407 USA (e-mail: radic@ece.ucsd.edu).

C. McKinstrie is with Bell Laboratories, Holmdel, NJ 07733 USA.

Digital Object Identifier 10.1109/LPT.2006.885591 guidance far from $1550 \mathrm{~nm}$. In contrast, silica photonic crystal fiber $(\mathrm{PCF})$ can, in principle, simultaneously guide spectrally distant signal, pump, and idler bands from 400 to $2500 \mathrm{~nm}$. The PCF transverse structure facilitates the nearly arbitrary dispersion profile synthesis required for phase matching between distant signal and pump waves [3], [4]. More importantly, rigorous dispersion control can be combined with high-confinement, providing an elevated effective nonlinear response $(\gamma)$. Sharping [5] has used these unique characteristics in the construction of an optical parametric oscillator operating in the pulsed regime. Andersen [6] has recently demonstrated continuous-wave (CW) conversion of high-power multimode $1493 \mathrm{~nm}$ and single-mode 1549-nm signal in PCF using a tunable Ti : Sapphire pump.

Until recently [7], no attempt had been made to explore the feasibility of distant, modulated signal translation from the conventional 1550-nm band to the visible band. PCF with dual zero-dispersion wavelengths (ZDWs) is a natural choice of medium, because of its ability to phase match commercially available (800- to $900-\mathrm{nm}$ ) pumps, $1550-\mathrm{nm}$ signal, and visible idler bands. This letter demonstrates, for the first time, the translation of single and wavelength-division-multiplexed (WDM) channels from 1550- to 500-nm band. Signals in the 1550-nm band were modulated, using both a harmonic pattern and a nonreturn-to-zero pseudorandom bit sequence (PRBS), translated parametrically and received in the 500-nm band. Translated WDM channels were demultiplexed and received separately, indicating no significant crosstalk. These results show that the standard telecommunication band can be translated to any position within the visible window by tuning either the pump or the signal band.

\section{EXPERIMENT AND DISCUSSION}

The experiment used standard PCF designed for supercontinuum generation in the $800-\mathrm{nm}$ spectral band. As a consequence, both signal $(1550 \mathrm{~nm})$ and idler $(500 \mathrm{~nm})$ bands are characterized by excessive loss which limits the power efficiency of the translator. The PCF phase matching contour is illustrated in Fig. 1, with the corresponding dispersion profile shown in the inset. The contour defines points at which signal (1550-nm band), pump (800-nm band), and idler (500-nm band) are strictly phase matched. In practical terms, the pump positioned near $800 \mathrm{~nm}$ allows the standard telecommunication $1550-\mathrm{nm}$ band to be mapped to the visible band idler while preserving the signal-pump-idler phase-matching condition.

The spectrally distant, degenerate parametric process was modeled by the standard set of coupled mode equations [1], which included the effect of pump-depletion. The translator efficiency was investigated as a function of signal and idler band losses, as illustrated in Fig. 2. For a lossless PCF, a $20-\mathrm{dBm}$ pump and $10-\mathrm{dBm}$ signal result in an idler power of $-7 \mathrm{dBm}$. A moderate $50-\mathrm{dB} / \mathrm{km}$ loss across all three bands 


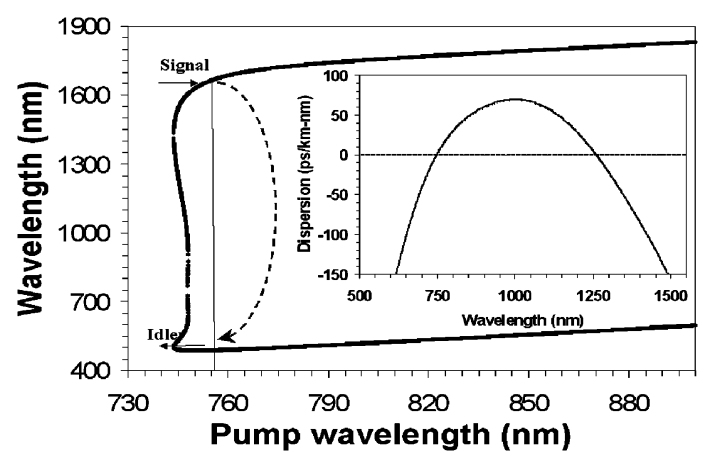

Fig. 1. PCF phase matching contour defined by dual zero dispersion wavelength characteristic (inset).

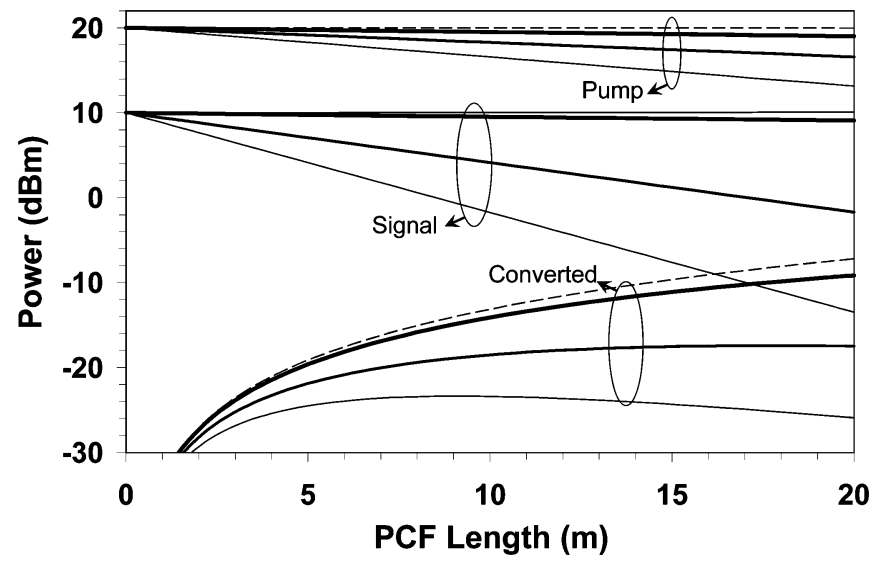

Fig. 2. Pump $(800 \mathrm{~nm})$, signal $(1550 \mathrm{~nm})$, and idler $(539 \mathrm{~nm})$ evolution in case of lossless (dashed curve) and lossy PCF. Corresponding losses for signal/pump/idler bands in decibels per kilometer: 50/50/50 (heavy curve), 600/175/200 (medium curve), and 1200/350/400 (thin curve).

which could be realized by a custom-drawn PCF would result in $2 \mathrm{~dB}$ lower idler power. Higher loss has a dramatic impact on the conversion efficiency, even for the short 20-m sample lengths used in this experiment.

A one-pump parametric device was constructed as shown in Fig. 3. A single-frequency CW Ti : Sapphire laser, tunable between 780 and $840 \mathrm{~nm}$ served as a parametric pump and operated in the anomalous dispersion region of the PCF, which was characterized by two ZDWs 750 and $1260 \mathrm{~nm}$, a maximual dispersion of $69.5 \mathrm{ps} / \mathrm{km} \cdot \mathrm{nm}$ at $1001 \mathrm{~nm}$, and an estimated nonlinear parameter of $70 \mathrm{~W}^{-1} \cdot \mathrm{km}^{-1}$ near the 800 -nm pump wavelength. The polarization-dependent nature of PCF required the use of a half-waveplate (W1) to align the pump polarization to a principal axis of the PCF. A dichroic beam splitter (M2) was used to combine the pump and signal (1550-nm) bands into the coupling objective (MO1). The first set of experiments was performed using a single external cavity laser signal source tuned from 1530 to $1600 \mathrm{~nm}$. The source was modulated using a Mach-Zehnder modulator driven by either a harmonic or a PRBS pattern generator with a $2^{7}-1$ or a $2^{31}-1$ pattern length with varying channel rates. Subsequently, the modulated signal was boosted (A) and filtered (F) in order to reject excess amplified spontaneous emission from the EDFA. A fiber polarization controller (PC) was used to maximize the gradient-index lens (G1) coupling to the free-space signal arm (G1-M1). The

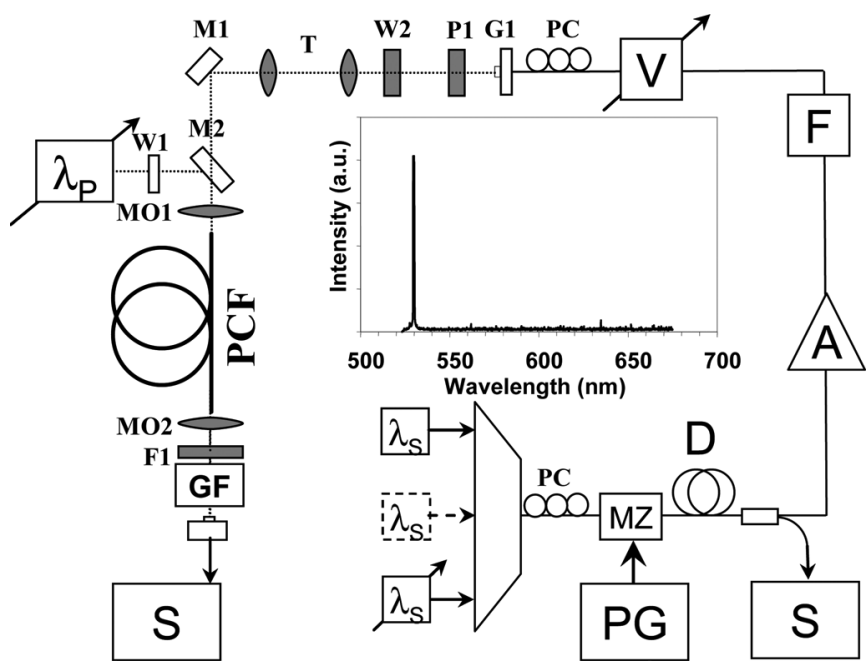

Fig. 3. Experimental setup; symbols as defined in text. Inset indicates typical visible (converted) single-channel spectrum. GF: Grating filter.

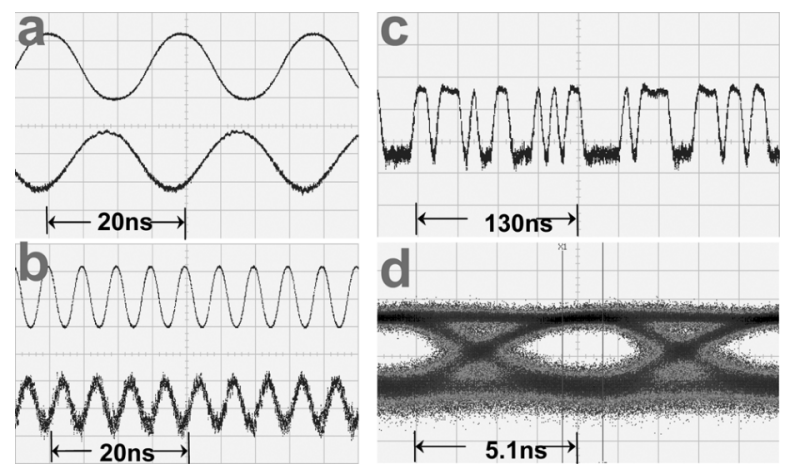

Fig. 4. (a) A 50-MHz harmonically modulated $1542-\mathrm{nm}$ signal (upper trace, 1550-nm PIN receiver) and its translated $(531 \mathrm{~nm}$ ) waveform (lower trace). (b) $200-\mathrm{MHz}$ harmonically modulated $1542-\mathrm{nm}$ signal (upper trace) and its translated waveform, corresponding to $3-\mathrm{dB}$ frequency rollover point of the visible receiver. (c) $155.52-\mathrm{MHz}$ modulated PRBS word received at $531 \mathrm{~nm}$. (d) 531-nm eye pattern. The eye penalty is dominated by the operation of the visible receiver beyond 3-dB rollover point.

combination of a linear polarizer (P1) and half-wave plate (W2) was used to align pump and signal polarization prior to insertion in the PCF, with minimal coupled powers of 120 and $10 \mathrm{~mW}$, respectively, throughout the experiment. A dedicated telescope (T) segment collimated the signal beam before it was coupled into the PCF objective (MO1).

The PCF output was collimated using a second objective (MO2) and filtered by a 600-nm low-pass dichroic element (F1), which separated the idler band from the pump and the signal bands. The final (visible) idler had sufficient power in all experimental configurations (single channel and WDM) to overcome the output coupling losses (MO2, F1, GF) and was well above the sensitivity threshold of the receiver. In the second set of experiments, the single tunable source was replaced by four WDM channels, which were modulated and decorrelated prior to the booster (A). In both sets of experiments, the input signal channels were monitored (C) prior to the booster stage using a fast oscilloscope (S).

Fig. 3 illustrates a typical spectrum obtained by a visible spectrometer with a resolution of $0.5 \mathrm{~nm}$. The idler was tuned 


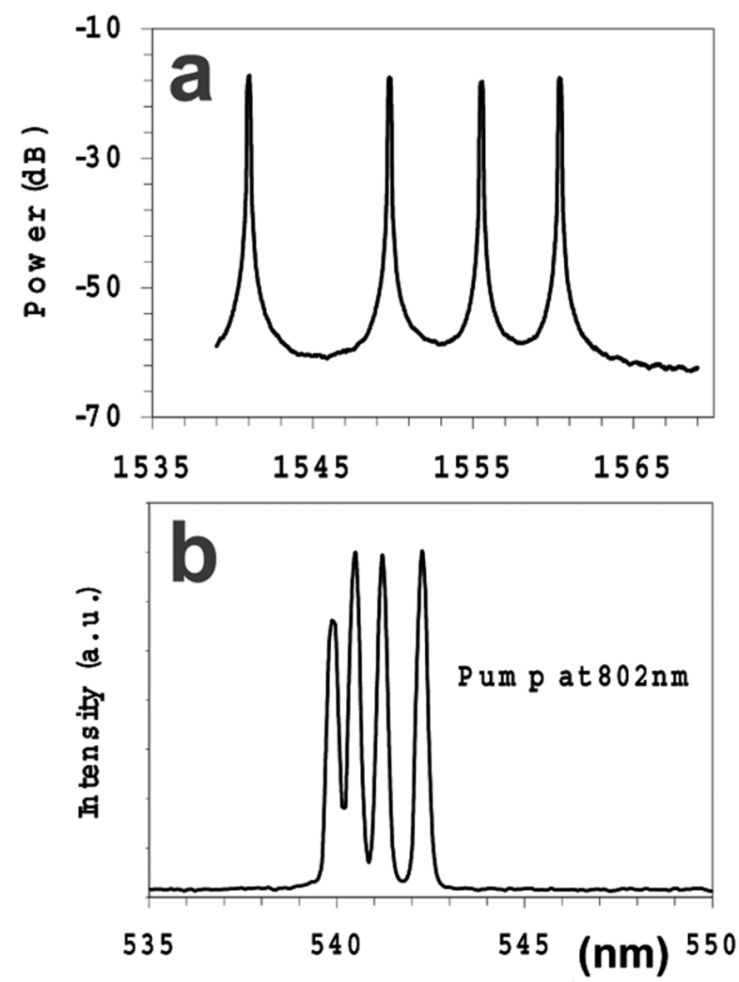

Fig. 5. Four WDM channels at $1541,1549,1555$, and $1560 \mathrm{~nm}$ are translated to visible spectral domain. (a) Signal and (b) idler band.

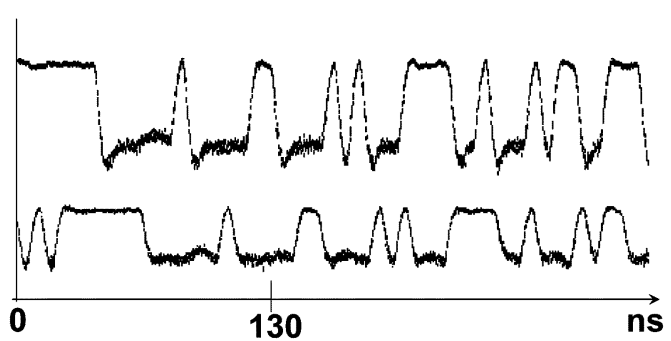

Fig. 6. Channels demultiplexed after visible translation: 574 (upper trace) and $576 \mathrm{~nm}$ (lower trace).

within the range $500-600 \mathrm{~nm}$ to demonstrate the available translator bandwidth, in excellent agreement with the estimated PCF phase-matching range.

The experiment used two PCF coils 18 and $20 \mathrm{~m}$ long and drawn from the same segment of fiber. The first coil allowed continuous tuning within the ranges of 515-525 and $530-542 \mathrm{~nm}$, whereas the second coil allowed tuning within $515-525$ and $565-580 \mathrm{~nm}$, which indicates slightly different dispersion characteristics.

The first modulation measurement was performed with the pump and signal positioned at 790 and $1541 \mathrm{~nm}$, respectively. The resulting idler waveform at $531 \mathrm{~nm}$ was measured using a standard visible detector (Hamamatsu C5331) with a cutoff frequency of $100 \mathrm{MHz}$. Although the signal was modulated at rates up to $10 \mathrm{GHz}$, the absence of a fast visible detector rendered any faster measurements $(>200 \mathrm{Mb} / \mathrm{s})$ impossible. The signal was modulated first using pure harmonic waveforms at 50 and $200 \mathrm{MHz}$, as shown in Fig. 4(a) and (b). The excessive distortion at $200 \mathrm{MHz}$ was induced by the rollover of the detector response. The measured idler power scaled linearly with the signal power, as expected for a nondepleted one-pump parametric interaction [8]. The second modulation measurement was performed using $155.52-\mathrm{Mb} / \mathrm{s}$ (OC-3) PRBS pattern, as shown in Fig. 4(c) and (d). The detected idler at a wavelength of 531 had a typical measured $Q$-factor of 5.76. This translated performance incorporates penalty mechanisms associated with the operation of the visible receiver beyond 3-dB rollover point, the noisy Ti: Sapphire pump, and excessive PCF loss at $1550 \mathrm{~nm}$. The last impairment dominated the link budget: the commercial PCF coils used in this experiment were designed for supercontinuum generation within the 800 - to $900-\mathrm{nm}$ range, and were not optimized for simultaneous single-mode transmission at 500, 800, and $1550 \mathrm{~nm}$. Consequently, the measured signal loss at $1550 \mathrm{~nm}$ (in absence of 800-nm pump) exceeded $15 \mathrm{~dB}$, corresponding to the high-loss model in Fig. 2, in excellent agreement with measured idler power of $-26 \mathrm{dBm}$. A set of bit-error-rate (BER) tests was performed using short and long word lengths: when $2^{7}-1$ PRBS is used, error-free BER $\left(<10^{-9}\right)$ was measured. However, when a $2^{31}-1$ was used, the measured BER degraded to $6 \times 10^{-8}$. This degradation is attributed to the nonuniform frequency response of the detector which leads to pattern-dependent errors within long pattern words.

In addition to the translation of a single modulated channel at $1550 \mathrm{~nm}$, we explored the feasibility of WDM (banded) translation. Four channels positioned at 1541, 1549, 1555, and 1560 were translated using a fixed and tunable Ti:Sapphire pump. Fig. 5 illustrates WDM band-casting for pump positioned $802 \mathrm{~nm}$. In the final experiment, two channels were spectrally demultiplexed, as illustrated in Fig. 6. The individual channel was demultiplexed using a grating filter in order to measure BER of $10^{-9}$. The measurement indicates no significant crosstalk, in agreement with the modeled prediction.

In conclusion, $375-\mathrm{THz}$ frequency translation of a single channel and WDM channels was demonstrated using a one-pump parametric interaction in a silica PCF. Signals in the telecommunication band were modulated, using harmonic and pseudorandom patterns, translated and recovered in the visible band. No significant impairment associated with the translation process was observed in the single- or multiple-channel case.

\section{REFERENCES}

[1] C. J. McKinstrie, S. Radic, and A. R. Chraplyvy, "Parametric amplifiers driven by two pump waves," IEEE Sel. Topics Quantum Electron., vol. 8, no. 3, pp. 538-538, May/Jun. 2002.

[2] J. Hansryd and P. Andreksen, "Broad-band continuous-wave-pumped fiber optical parametric amplifier with 49-dB gain and wavelength-conversion efficiency," IEEE Photon. Technol. Lett., vol. 13, no. 3, pp. 194-196, Mar. 2001.

[3] P. Russell, "Photonic crystal fibers," Science, vol. 299, pp. 358-362, 2003.

[4] A. Y. H. Chen, G. L. Wong, S. G. Murdoch, R. Leonhardt, J. D. Harvey, J. C. Knight, W. J. Wadsworth, and P. St. J. Russell, "Widely tunable optical parametric generation in a PCF," Opt. Lett., vol. 30, pp. $762-762,2005$.

[5] J. Sharping, M. Fiorentino, P. Kumar, and R. Windeler, "Optical parametric oscillator based on four-wave mixing in microstructure fiber," Opt. Lett., vol. 27, pp. 1675-1675, 2002.

[6] T. V. Andersen, K. M. Hilligsøe, C. K. Nielsen, J. Thøgersen, K. P. Hansen, S. R. Keiding, and J. J. Larsen, "Continuous-wave wavelength conversion in a photonic crystal fiber with two ZDW," Opt. Express, vol. 12, pp. 4114-4122, 2004.

[7] R. Jiang, R. Saperstein, N. Alic, M. Nezhad, C. McKinstrie, J. Ford, Y. Fainman, and S. Radic, "375 THz parametric translation of modulated signal from $1550 \mathrm{~nm}$ to visible band," presented at the Optical Fiber Conf., Anaheim, CA, 2006, Postdeadlne paper PD16. 